\title{
Genetics of bipolar disorder
}

This article was published in the following Dove Press journal:

The Application of Clinical Genetics

12 February 2014

Number of times this article has been viewed

\section{Berit Kerner}

Semel Institute for Neuroscience and Human Behavior, University of California, Los Angeles, Los Angeles, CA, USA
Correspondence: Berit Kerner Semel Institute for Neuroscience and Human Behavior, University of California, Los Angeles, 760 Westwood Plaza, Los Angeles, CA 90095-I76I, USA

Tel +l 310 39l 5599

Fax +l 310 391 5599

Email bkerner@mednet.ucla.edu
Abstract: Bipolar disorder is a common, complex genetic disorder, but the mode of transmission remains to be discovered. Many researchers assume that common genomic variants carry some risk for manifesting the disease. The research community has celebrated the first genome-wide significant associations between common single nucleotide polymorphisms (SNPs) and bipolar disorder. Currently, attempts are under way to translate these findings into clinical practice, genetic counseling, and predictive testing. However, some experts remain cautious. After all, common variants explain only a very small percentage of the genetic risk, and functional consequences of the discovered SNPs are inconclusive. Furthermore, the associated SNPs are not disease specific, and the majority of individuals with a "risk" allele are healthy. On the other hand, population-based genome-wide studies in psychiatric disorders have rediscovered rare structural variants and mutations in genes, which were previously known to cause genetic syndromes and monogenic Mendelian disorders. In many Mendelian syndromes, psychiatric symptoms are prevalent. Although these conditions do not fit the classic description of any specific psychiatric disorder, they often show nonspecific psychiatric symptoms that cross diagnostic boundaries, including intellectual disability, behavioral abnormalities, mood disorders, anxiety disorders, attention deficit, impulse control deficit, and psychosis. Although testing for chromosomal disorders and monogenic Mendelian disorders is well established, testing for common variants is still controversial. The standard concept of genetic testing includes at least three broad criteria that need to be fulfilled before new genetic tests should be introduced: analytical validity, clinical validity, and clinical utility. These criteria are currently not fulfilled for common genomic variants in psychiatric disorders. Further work is clearly needed before genetic testing for common variants in psychiatric disorders should be established.

Keywords: bipolar disorder, risk factors, genomic variants, structural variants, Mendelian disorders, genetic testing

\section{Introduction}

Bipolar disorder is a severe and common mental disorder. It is present in approximately 5.7 million American adults, or 2.6 percent of the US population aged 18 years and older in any given year. ${ }^{1}$ At the core of the disease are dramatic and unpredictable mood swings between mania and depression. The diagnosis is usually made based on a combination of clinical indicators from a list of diagnostic criteria. ${ }^{2}$ Bipolar disorder has a characteristic disease course, but the individual symptoms of bipolar disorder are not specific, and they may vary considerably from person to person and over the disease course. In some individuals, symptoms of depression prevail; in others, the clinical presentation is dominated by elevated or irritable mood with excessive energy, hyperactivity, and even aggressiveness. ${ }^{3}$ About half of the individuals diagnosed with bipolar disorder 
also suffer from distorted experiences of reality, known as hallucinations and delusions. ${ }^{4}$ Because the symptoms of bipolar disorder are shared with many psychiatric disorders, the diagnostic boundaries are not clearly defined. Not uncommonly, patients change diagnoses over the course of a lifetime. The clinical presentation is highly variable; hence, bipolar disorder has also been conceptualized as a group of related mood disorders, referred to as bipolar spectrum disorders. In addition, anxiety disorders, abuse of illegal substances, alcohol dependence, and attention-deficit/hyperactivity disorder often co-occur with bipolar disorder. ${ }^{5-8}$ This phenomenon is not well understood. Although some experts believe that these conditions share common genetic risk factors with bipolar disorder, others have been more cautious. The disease onset of bipolar disorder is during adolescence and early adulthood, but the diagnosis is often delayed by many years. ${ }^{9}$ A contributing factor is the complex clinical picture, with sometimes very subtle symptoms, at disease onset. This is particularly tragic, as about half of the individuals with bipolar disorder attempt suicide at least once in their lifetime, and many complete the attempt. ${ }^{10,11}$ Despite severe symptoms, treatment can be successful if the correct diagnosis is made and treatment is initiated early. ${ }^{12}$ Consequently, enormous efforts have been made to identify genetic risk factors or biomarkers that would identify individuals at risk and could facilitate early diagnosis and treatment.

\section{Bipolar as a common complex disorder}

Bipolar disorder is a complex and multifactorial disorder. The heritability of bipolar disorder based on concordance rates for bipolar disorder in twin studies has been estimated to be between $60 \%$ and $80 \% .{ }^{13}$ Slightly lower estimates of genetic risk have been suggested based on family studies and large population cohorts. ${ }^{14}$ Even though this evidence for genetic risk factors is convincing, most clinicians would agree that a positive family history of bipolar disorder is actually not very common in everyday clinical practice. In fact, it is quite rare to find families in which bipolar disorder affects multiple members over several generations, as would be expected for a monogenic Mendelian disorder. Many patients are isolated cases. In addition to genetic risk factors, nongenetic risk factors might contribute to the manifestation of bipolar disorder, as well, such as alcohol and drug dependence or physical and sexual abuse. ${ }^{15}$ It has been well established that environmental and social risk factors play a significant role in schizophrenia. ${ }^{16-19}$ A similar picture might evolve in bipolar disorder, as well. ${ }^{20}$
In addition to inherited genomic variants, recent evidence supports a significant role of de novo protein-damaging mutations in psychiatric disorders. ${ }^{21}$ Experts in the field agree that susceptibility to bipolar disorder is most likely influenced by many genetic risk factors with small to moderate effect. Individual-specific and family-specific environmental factors might play a role, as well. The results of genome-wide association studies have supported this disease model. In very large population-based studies of thousands of individuals, a handful of replicated association signals have emerged at the level of genome-wide statistical significance. ${ }^{22}$ In general, these variants have had very small effect sizes. Due to space constraints, I shall focus only on single nucleotide polymorphisms (SNPs) in the genes $C A C N A 1 C, O D Z 4$, and $N C A N$, which have emerged as promising candidate genes for bipolar disorder in genomewide association studies. I admit that this selection could be disputed, but it is beyond the scope of this review to mention all candidate genes for bipolar disorder that have emerged so far. As no gene could be considered as an undeniable risk factor, the interested reader is referred to other recent reviews on this subject. ${ }^{23}$ On the other hand, rare genetic syndromes and monogenic Mendelian disorders could present with psychiatric symptoms that closely resemble bipolar disorder or schizophrenia. These genetic disorders are often not properly diagnosed (Figure 1). Therefore, it is important to alert clinicians to genetic syndromes that could resemble primary psychiatric disorders. In many cases, the genetic

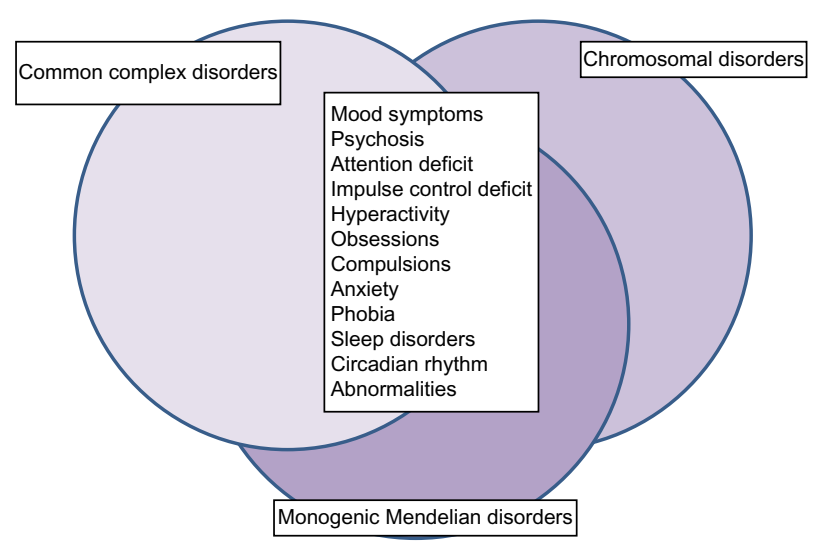

Figure I Symptoms of mood disorders are shared among common complex disorders, rare chromosomal disorders, and monogenic Mendelian disorders. Although many common complex disorders and rare Mendelian disorders share psychiatric symptoms, they do not always share the same genetic risk factors. Common complex disorders, such as bipolar disorder, are likely influenced by many genetic variants with small effect, in addition to environmental risk factors. Rare chromosomal disorders are characterized by large chromosomal deletions and duplications, which could potentially affect hundreds of genes. Rare monogenic Mendelian disorders are caused by characteristic mutations in a single gene. These differences in genetic risk factors have important consequences for risk prediction, genetic testing, and counseling. 
cause is already known, and genetic testing could assist in the differential diagnosis.

\section{Common variants as risk factors for bipolar disorder CACNA I C (calcium channel, voltage- dependent, $L$ type, alpha- I $C$ subunit)}

The SNP rs1006737 in the gene CACNA1C is the most replicated and most studied common genomic variant associated with bipolar disorder to date. ${ }^{24-26}$ The SNP is located in an intronic region and it occurs with significant allele frequency differences in all ethnic populations. The A allele, which is thought to be a risk factor for bipolar disorder, is present in $31 \%$ of European populations and in only $6 \%$ of Asian populations, but in almost $56 \%$ of individuals of African descent. As a result of these differences in allele frequencies, this SNP is vulnerable to confounding effects of ethnic admixture in genome-wide association studies. In fact, the association between the A allele of rs1006737 and bipolar disorder, first reported in a Caucasian population, could not be replicated with genome-wide significance in individuals of African descent or in some studies in Europe and Asia. ${ }^{27-29}$ Although the A allele seems to increase the risk of bipolar disorder in some population subgroups, most individuals who carry the "minor" allele are healthy. Therefore, the question remains how rs 1006737 could influence disease processes in bipolar disorder. As the SNP is not located in the coding region of the gene $C A C N A 1 C$, researchers have hypothesized that the variant could influence gene expression. In postmortem brain studies, scientists found evidence of reduced $C A C N A 1 C$ gene expression in individuals with the A allele, particularly in the cerebellum but not in other brain regions. ${ }^{30}$ However, this finding could not be replicated by other researchers, and some groups have even found increased gene expression. ${ }^{31}$ Further research is clearly needed to explain these discrepancies before definite conclusions can be reached.

Investigators have studied functional impairment in individuals with bipolar disorder who carried the A allele, particularly in the domains of executive function and emotional face processing. ${ }^{32,33}$ Although some researchers have found no effect, others reported significant reduction in cognitive function, but only in individuals who carried two A alleles, compared with patients with only one or no A allele. ${ }^{34}$ Several groups could not replicate these results or even reported effects in the opposite direction. ${ }^{35,36}$ The results in patients with bipolar disorder have been inconclusive. Despite these discrepancies, studies generally agree that significant effects have not been observed in healthy individuals. Several factors could have contributed to the conflicting results, including small effect sizes and small sample sizes, in addition to differences in methodology, analysis, and interpretation across studies. Additional complexity has been added to the debate after researchers discovered a significant association signal with rs 1006737 in a study in which patients with major affective disorder, bipolar disorder, autism spectrum disorder, attention-deficit/hyperactivity disorder, and schizophrenia had been combined. This finding indicated a lack of clinical specificity. ${ }^{37}$ Recently, studies have claimed additional intronic SNPs in and around CACNAIC as being associated with psychiatric disorders, but replication and functional studies are still lacking. ${ }^{38,39}$

Psychiatric genetics is rooted in the assumption that common genomic variants tag functional variants in or around the gene closest to the common variant. Therefore, it is often implied that the closest gene might have some functional role in the disease processes of the associated disorder. CACNA1C is a large gene with over 11,541 known variants. Most of these variants are located in the introns and regions downstream of the gene. Missense mutations in exons are very rare. $C A C N A 1 C$ codes for the alpha- 1 subunit of a voltage-dependent calcium channel. This subunit forms a transmembrane channel through which calcium ions enter the cell. Calcium channels are important neuronal regulators of muscle contraction in the heart, but they are also involved in skeletal muscle contraction. In the brain, CACNA1C could be involved in axon guidance and synaptic transmission. Mutations in the coding region of CACNA1C are the cause of Timothy syndrome (Mendelian Inheritance in Man [MIM] \#601005), a rare Mendelian disorder, also known as long QT syndrome with syndactyly. ${ }^{40-42}$

Timothy syndrome is a multiorgan disease with lethal arrhythmias and congenital heart disease, developmental abnormalities of fingers and toes, immune deficiency, intermittent hypoglycemia, cognitive impairment, and behavioral abnormalities resembling autism. The syndrome is caused by one of two known heterozygote mutations (p.Gly402Ser and p.Gly406Arg) in exon 8 of the gene. This functional region encodes the transmembrane calcium channel. The mutated codon is a key regulator of calcium transport across the cell membrane. If one of these mutations is present, increased calcium influx into the cell occurs, resulting in increased excitability of the cell. Because of these severe consequences, children with Timothy syndrome often die at the age of 2 or 3 years. Survival into adulthood is very rare. However, a milder form of the disorder, Brugada syndrome type 3 
(MIM \#611875), has been described. ${ }^{43}$ Brugada syndrome is characterized by cardiac arrhythmia with characteristic electrocardiogram changes. Sudden death could occur due to ventricular fibrillation, even though most individuals with this syndrome survive into adulthood. The disorder is caused by heterozygote mutations in different functional regions of CACNA1C (Gly490Arg in exon 10 and Ala39Val in exon 2). Current knowledge suggests that mutations in the coding region of the gene $C A C N A 1 C$ do not lead to bipolar disorder but rather to a monogenic Mendelian disorder with cognitive disability and autistic features.

In mice, homozygous mutations that completely eliminate the CACNA1C protein are lethal. Mutations that impaired the protein function resulted in reduced insulin secretion, glucose intolerance, poor motor coordination, increased anxiety, and hypoactivity in some studies. ${ }^{44}$ In more than 69 papers, researchers have explored the relationship between rs1006737 and bipolar disorder, as well as other psychiatric disorders. ${ }^{45}$ However, further work is clearly needed before the SNP rs1006737 could be established as an undisputed genetic risk factor for bipolar disorder.

\section{ODZ4 (teneurin transmembrane protein 4)}

The common variant rs12576775 in the intron of gene ODZ4 has been associated with bipolar disorder. However, significant association has also been established with autism spectrum disorders, attention-deficit/hyperactivity disorder, major depressive disorder, and schizophrenia in a combined analysis of these psychiatric disorders. ${ }^{22,37,38,46}$ The variant has a minor allele frequency of about $10 \%$ across all ethnic populations with the exception of European populations, in which the minor allele occurs in about $20 \%$ of individuals. Researchers have studied the effect of rs 12576775 in individuals with bipolar disorder and also in healthy individuals using functional and structural brain imaging; however, the results of these studies have been inconclusive. ${ }^{47-50}$

ODZ4 is a large transmembrane protein and its structure resembles signal transduction molecules. ${ }^{51}$ During brain development, ODZ4 appears to play a central role in the regulation of neuronal and synaptic connectivity. ${ }^{52}$ At later stages of brain maturation, ODZ4 has been shown to orchestrate the development and differentiation of oligodendrocytes and the myelination of neuronal axons. ${ }^{53}$ The gene has 14,410 known variants, but mutations in the coding region of the gene are not known to cause a Mendelian disorder. Knowledge about ODZ4 is still very limited, and more functional studies are clearly needed before clinical applications could be considered.

\section{NCAN (neurocan)}

$N C A N$ is a large gene located on chromosome 19p13.11. The SNP rs1064395, which is found in an intronic region of the gene, has been associated with bipolar disorder in one study, ${ }^{54}$ but so far not all studies have replicated this finding. ${ }^{55}$ Even though association between variants in NCAN and schizophrenia has been tested, no genome-wide significant results have been found. ${ }^{56} \mathrm{Rs} 1064395$ affects only one of five alternative transcripts of the gene. Significant differences in allele frequencies of the associated allele have been observed across all ethnic populations. Although the overall frequency of the A allele (or disease-associated allele) is about 23\%, it is the major allele in $51 \%$ of African populations. The disease-associated allele is present in only $12 \%$ of individuals in Asia and in 15\% of individuals in Europe. Studies in postmortem brains of individuals with bipolar disorder or schizophrenia found increased cortical folding in some brain regions of patients with the A allele compared with healthy controls; however, no effect of the A allele was detected in the comparison of healthy individuals with the A allele and healthy individuals with the alternative allele. ${ }^{57}$

The gene $N C A N$ codes for a large secreted protein that is found predominantly in the extracellular space, the lumen of the Golgi apparatus, and the lysosomal cavities. Public databases list 741 known variants in the gene, but coding variants have not been associated with any Mendelian disorder. The protein is involved in the modulation of cell adhesion, cell migration, and axon guidance. However, knockout mice had normal brain structure and function. Mild deficits in synaptic plasticity in neurons of the hippocampus have been observed, ${ }^{58}$ but only the complete knockout of four related proteoglycans resulted in severe structural and functional abnormalities. ${ }^{59}$ These findings indicate that alternative mechanisms might exist to compensate for the loss of function of the NCAN protein.

Associations with genomic variants in $N C A N$ do not appear to be disease specific. SNPs close to the gene have been associated with abnormalities in lipid metabolism at the genome-wide level of statistical significance in various ethnic populations; ${ }^{60-64}$ however, negative results have also been reported. ${ }^{65}$

The question remains how to interpret statistically significant association signals with common genomic variants and how to translate the results into clinical practice. It remains to be seen to what degree statistically significant association signals indicate genetic risk factors and how they could explain disease processes leading to bipolar disorder. Based on current knowledge, any genetic testing for bipolar disorder 
involving common genetic variants lacks scientific support and is clearly premature.

\section{Chromosomal disorders with symptoms of bipolar disorder}

Most clinicians would agree that bipolar disorder is a complex and multifactorial disorder with a relatively low recurrence risk. Psychiatric symptoms, however, are also common in certain rare monogenic Mendelian disorders and chromosomal disorders, also known as duplication and deletion syndromes. Even though these diseases do not meet the classic description of bipolar disorder or schizophrenia, they could present with acute symptoms of mania, severe depression, or psychosis; therefore, the differential diagnosis could be challenging. Even though these conditions are individually very rare, together they present a nonignorable fraction of cases with psychiatric symptoms. The Online Mendelian Inheritance in Man (OMIM) database lists about 88 entries with mood symptoms, and about 64 of these conditions are chromosomal disorders. ${ }^{66}$ Psychosis in Mendelian disorders is even more common than extreme mood symptoms. In fact, symptoms of hallucinations and delusions are described in about 138 entries in OMIM, and 93 of these are chromosomal disorders. Some of these disorders are rare dominant Mendelian conditions or structural abnormalities shared with a parent. Under these circumstances, the recurrence risk could be as high as $50 \%$. Therefore, it is essential to consider these disorders in the differential diagnosis of bipolar disorder or schizophrenia so that the correct diagnosis can be established and the families counseled about the increased recurrence risk. Although it is beyond the scope of this review to cover this topic comprehensively, I will present four examples of chromosomal disorders and one example of a rare Mendelian disorder in which mood symptoms and psychosis are part of the clinical presentation (Table 1).

Table I Phenotype comparison of four rare chromosomal disorders and one monogenic Mendelian disorder reveals strong similarities and overlapping psychiatric symptoms ${ }^{\mathrm{a}}$

\begin{tabular}{|c|c|c|c|c|c|}
\hline \multirow[t]{2}{*}{ Genetic abnormality } & \multicolumn{3}{|l|}{ PWS } & \multirow{2}{*}{$\frac{\text { VCF }}{\text { del 22qII }}$} & \multirow{2}{*}{$\frac{\text { SMS }}{\text { RAII (I7p II.2) }}$} \\
\hline & del I5qII & del I5qI3.3 & del 10q26 & & \\
\hline OMIM & $\# 176270$ & $\# 612001$ & $\# 609625$ & $\# 192430$ & $\# 182290$ \\
\hline Depression & $\mathrm{x}$ & $\mathrm{x}$ & & $\mathrm{x}$ & $\mathrm{x}$ \\
\hline Rapid mood swings & $x$ & $x$ & $x$ & $x$ & $x$ \\
\hline Irritability & $x$ & & & & $x$ \\
\hline Hyperactivity & & $x$ & $x$ & $x$ & $x$ \\
\hline Impulsivity & $x$ & $x$ & & & $x$ \\
\hline Aggressiveness & $x$ & $x$ & $\mathrm{x}$ & & $x$ \\
\hline Self-injurious behavior & & & & & $x$ \\
\hline Sleep disorder & & & & & $x$ \\
\hline Circadian abnormalities & & & & & $x$ \\
\hline Attention deficit & $x$ & $x$ & $x$ & $x$ & $x$ \\
\hline OCD & $x$ & $x$ & & $x$ & $x$ \\
\hline Anxiety disorders & & $x$ & & $x$ & \\
\hline Psychosis & $x$ & $x$ & & $x$ & \\
\hline Learning disability & & & $x$ & $x$ & $x$ \\
\hline Mild intellectual disability & $x$ & $x$ & $x$ & $x$ & $x$ \\
\hline Moderate intellectual disability & $x$ & $x$ & $x$ & $x$ & $x$ \\
\hline Severe intellectual disability & & $x$ & $x$ & $x$ & \\
\hline Language delay & $x$ & & $x$ & $\mathrm{x}$ & $\mathrm{x}$ \\
\hline Autistic features & $x$ & $x$ & $x$ & $x$ & \\
\hline Seizure disorders & $x$ & $x$ & & & $x$ \\
\hline Obesity & $x$ & & & & $x$ \\
\hline Cardiac abnormalities & & $x$ & $x$ & $x$ & \\
\hline Urogenital abnormalities & & & $x$ & & \\
\hline Endocrine abnormalities & $x$ & & & $x$ & \\
\hline Skeletal abnormalities & $x$ & $x$ & $x$ & & $x$ \\
\hline Facial abnormalities & $x$ & x & $x$ & $x$ & $x$ \\
\hline Frequency & $\mathrm{I}: 22,000$ & $\mathrm{I}: 22,000$ & Unknown & $1: 2,000$ & $\mathrm{I}: 15,000$ \\
\hline
\end{tabular}

Notes: a Chromosomal deletion syndromes and monogenic Mendelian disorders share many symptoms with psychiatric disorders, even though the disease course is not specific for any one single disorder. The presence of intellectual disability, facial abnormalities, and neurologic symptoms could indicate a genetic syndrome rather than a common complex psychiatric disorder.

Abbreviations: OCD, obsessive-compulsive disorder; OMIM, Online Mendelian Inheritance in Man; PWS, Prader-Willi syndrome; RAI, retinoic acid-induced I gene; SMS, Smith-Magenis syndrome; VCF, velo-cardio-facial syndrome. 


\section{Prader-Willi syndrome (MIM \#I 76270) (chromosome I5q I I deletion syndrome)}

Prader-Willi syndrome is a classic example of a chromosomal disorder with prominent mood symptoms and psychosis. This syndrome, also known as chromosome $15 \mathrm{q} 11$ deletion syndrome, is characterized by obesity, small hands and feet, characteristic facial features, and mild to moderate intellectual disability. Children with Prader-Willi syndrome develop severe mood disorder with frequent mood swings, irritability, and aggressive outbursts, as well as obsessive-compulsive behavior. ${ }^{67}$ Attention deficit, autism, and language delay are also commonly observed. ${ }^{68,69}$ During early adulthood, hallucinations and delusions occur in almost $30 \%$ of cases while mood symptoms persist. Seizure disorders are not uncommon in Prader-Willi syndrome. ${ }^{70}$ The syndrome is caused by an imbalance between maternal and paternal genetic material on chromosome 15q11. These imbalances could result from a small deletion on the paternal chromosome, which could vary considerably in size. The smallest deletion described in the literature removed a differentially methylated $5^{\prime}$ regulatory exon of the gene SNRPN (small nuclear ribonucleoprotein polypeptide N), which changed the methylation pattern and, consequently, the gene expression levels of hundreds of genes. ${ }^{71}$ Less commonly, a duplication of maternal material is found. Even though most cases develop spontaneously, familial transmission has been observed, and transmitting mothers are asymptomatic. ${ }^{72}$ The syndrome occurs with a prevalence of about one in 22,000 worldwide. The chromosomal changes in this syndrome are disease specific and highly penetrant. If the clinical presentation suggests Prader-Willi syndrome, genetic testing and counseling are recommended.

\section{Chromosome I5qI3.3 deletion syndrome (MIM \#6 I200I)}

Adjacent to the Prader-Willi region, a large two megabase deletion on chromosome 15q13.3 has been described. Patients with this chromosomal abnormality have mild to moderate intellectual disability and mild dysmorphic features of the hands and face. ${ }^{73,74}$ Frequently, carriers of the deletion develop seizure disorders and autistic traits. ${ }^{75-77}$ The clinical presentation of individuals with the $15 \mathrm{q} 13.3$ deletion syndrome is highly variable, ranging from asymptomatic to severe intellectual impairment. ${ }^{78}$ Behavioral abnormalities are common and include aggressiveness, impulse control problems, attention deficits, and hyperactivity. ${ }^{79,80}$ Individuals are sometimes misdiagnosed with bipolar disorder or obsessive-compulsive disorder. Anxiety disorders and phobias have also been described. Psychotic symptoms could lead to the diagnosis of schizophrenia in some cases, even though the syndromic gestalt of the disorder does not fit classical descriptions of schizophrenia. ${ }^{81,82}$ Increasing evidence suggests that reduced function of the gene CHRNA7 is responsible for the neuropsychiatric deficits in this deletion syndrome. ${ }^{83,84}$ Carriers of deletions involving the gene CHRNA7 have severe mental disability, seizure disorders, and low muscle tone. ${ }^{85}$ On brain imaging studies, mild hypogenesis of the corpus callosum has been detected. The $15 \mathrm{q} 13.3$ microdeletion is a contiguous gene deletion inherited in an autosomal dominant manner. Approximately $25 \%$ of 15 q13.3 microdeletions are de novo; approximately $75 \%$ are inherited ${ }^{86}$ Offspring of an individual with the $15 \mathrm{q} 13.3$ microdeletion have a $50 \%$ chance of inheriting the deletion. Although prenatal testing is technically feasible, it is not possible to reliably predict the phenotype based on the laboratory finding of a 15q13.3 microdeletion, because of reduced penetrance. Mutations on the nondeleted chromosome could have disease-modifying effects.

\section{Chromosome 10q26 deletion syndrome (MIM \#609625)}

The chromosome 10q26 deletion syndrome is characterized by mild to moderate intellectual disability, short stature, small head, and characteristic facial features. Cardiac, renal, and genital abnormalities have been described in some individuals ${ }^{87}$ Rapid mood swings are common: eg, very affectionate behavior could unpredictably turn into aggressive and provocative actions. ${ }^{88}$ Hyperactivity and attention deficits are also common. ${ }^{89,90}$ Poor speech and language development, as well as autistic traits, have been described. Cognitive impairment is highly variable and can range from mild learning disabilities to severe mental handicap and absent speech. Familial transmission has been described, ${ }^{91}$ and in these instances the risk could be as high as $50 \%$.

\section{Velo-cardio-facial syndrome (MIM \#602054) (chromosome 22ql I deletion syndrome)}

Velo-cardio-facial syndrome is caused by a three megabase deletion on chromosome 22q11 spanning about 40 genes. The deletion has a population prevalence of about one in 2,000 . The syndrome is a multiorgan, complex disorder. The spectrum of symptoms is wide-ranging, from near normal to severe impairment and even life-threatening manifestations. ${ }^{92}$ Familial transmission and intrafamilial variability have 
been reported. ${ }^{93}$ Psychiatric symptoms are common. Children and adolescents with velo-cardio-facial syndrome are at increased risk for depression, anxiety, and attentiondeficit/hyperactivity disorders. ${ }^{94,95}$ Obsessive-compulsive behavior and autistic features are also not uncommon. About $30 \%$ of individuals with the deletion develop behavioral symptoms that could resemble bipolar disorder, or even hallucinations and delusions. ${ }^{96,97}$ Some individuals have been diagnosed with schizophrenia because of intellectual decline after the onset of psychosis. ${ }^{98,99}$

\section{Monogenic disorders with symptoms of bipolar disorder}

Point mutations and other genomic changes in a single gene could change the structure and function of the encoded protein. Sometimes, the loss of a single gene can result in severe complex disorders, also known as monogenic Mendelian disorders. Familial transmission of these disorders is very rare in severely affected individuals with early onset, but it is not uncommon in milder cases or disorders with onset in adulthood.

\section{Smith-Magenis syndrome (MIM \#I 82290)}

Smith-Magenis syndrome is a genetic syndrome characterized by mild to moderate intellectual disability, self-injurious behaviors, obesity, skeletal abnormalities, and characteristic facial features. ${ }^{100}$ Sleep disturbances are common due to abnormal circadian rhythms. The syndrome could be caused by a microdeletion on chromosome $17 \mathrm{p} 11.2$, but most of the symptoms have been traced back to the loss of function of a single gene, RAII (retinoic acid induced 1). Small deletions, frameshift mutations, premature stop codons, and missense mutations in RAII could result in all the major features of the syndrome. ${ }^{101,102}$ Smith-Magenis syndrome affects approximately one in 25,000 individuals. ${ }^{103}$ Genetic testing for this disorder is well established, and genetic counseling is recommended.

\section{Summary}

In common complex disorders, genome-wide association studies have identified common variants that might indicate a small increase in genetic risk. Based on these results, the possibility of genetic testing has been discussed among clinicians, researchers, and patients alike. ${ }^{104}$ Although genetic testing is well established for chromosomal disorders or monogenic Mendelian disorder, the issue is more ambiguous in common complex multigenic conditions, due to the nature of the identified genomic variants. In the current debate, it might be advisable to learn from established principles and practices. The National Institutes of Health/Department of Energy Task Force on Genetic Testing has established three criteria that should be fulfilled before a new genetic test can be introduced, particularly in the context of genetic screening. ${ }^{105}$ These criteria are analytical validity, clinical validity, and clinical utility. Analytical validity refers to the accuracy and precision of a genetic test performed in a clinical laboratory. The second criterion, clinical validity, refers to a range of clinical performance measures, including clinical sensitivity, clinical specificity, and positive predictive value. The third requirement, clinical utility, refers to the usefulness of a test in clinical settings to improve the health of individuals.

Chromosomal disorders and monogenic Mendelian disorders with mood symptoms and psychosis are caused by rare structural genomic variants and protein-damaging mutations in single genes. Rare genetic mutations in these conditions are functional, disease specific, and highly penetrant. Even though these conditions are individually rare, they could still pose a nonignorable risk because the recurrence rate could be as high as $50 \%$ if the genetic risk factor is also present in one of the parents. As transmitting parents might be asymptomatic, genetic testing is recommended for every individual in whom mood symptoms are combined with neurological abnormalities or cognitive symptoms suggesting a Mendelian disorder or genetic syndrome.

Although genetic tests for Mendelian disorders and chromosomal disorders are well established, specific, and sensitive with high predictive value, the issue is more controversial for common complex disorders. After all, most individuals who carry the common "risk" allele are healthy and are not expected to develop the disease. In addition, the associated variants are usually not disease specific but have been associated with a wide range of disorders. Therefore, common variants are not suitable to predict the presence or absence of a clinical disease or the likelihood of developing a disease. In summary, the clinical utility of genetic tests for common complex disorders has not been clearly established, and the risk-benefit ratio remains unclear. Therefore, the risk of misinterpretation and misuse of genetic tests in common complex psychiatric disorders is high, especially if the tests are directly marketed to consumers. The potential for stigmatization, discrimination, anxiety, and burden on family relationships is real and should not be underestimated. As the field of psychiatric genetics advances, community interests, particularly regarding those who carry a "risk" allele - in some cases more than $50 \%$ of a specific ethnic 
population - should be assessed and risk-benefit ratios discussed. Clearly, more work is needed before genetic testing for common complex disorders can be established.

\section{Acknowledgment}

This work was supported by a grant from the National Institute of Mental Health R01MH085744-03 and a fellowship from the Brain and Behavior Research Foundation (NARSAD Young Investigator Grant). I am grateful to Jake Carpenter for editorial assistance.

\section{Disclosure}

The author reports no conflicts of interest in this work.

\section{References}

1. National Institute of Mental Health. Bipolar disorder among adults. Available from: http://www.nimh.nih.gov/statistics/1bipolar_adult.shtml. Accessed January 10, 2014.

2. American Psychiatric Association [homepage on the internet]. Available from: http://www.psychiatry.org/home. Accessed January 10, 2014

3. Merikangas KR, Cui L, Heaton L, et al. Independence of familial transmission of mania and depression: results of the NIMH family study of affective spectrum disorders. Mol Psychiatry. Epub October 15, 2013.

4. Vandeleur CL, Merikangas KR, Strippoli MP, Castelao E, Preisig M. Specificity of psychosis, mania and major depression in a contemporary family study. Mol Psychiatry. Epub October 15, 2013.

5. Corry J, Green M, Roberts G, et al. Anxiety, stress and perfectionism in bipolar disorder. J Affect Disord. 2013;151(3):1016-1024.

6. van Emmerik-van Oortmerssen K, van de Glind G, Koeter MW, et al. Psychiatric comorbidity in treatment seeking substance use disorder patients with and without ADHD; results of the IASP study. Addiction. Epub October 4, 2013.

7. Di Florio A, Craddock N, van den Bree M. Alcohol misuse in bipolar disorder. A systematic review and meta-analysis of comorbidity rates. Eur Psychiatry. Epub September 25, 2013.

8. Di Nicola M, Sala L, Romo L, et al. Adult attention-deficit/hyperactivity disorder in major depressed and bipolar subjects: role of personality traits and clinical implications. Eur Arch Psychiatry Clin Neurosci. Epub September 28, 2013.

9. Leboyer M, Kupfer DJ. Bipolar disorder: new perspectives in health care and prevention. J Clin Psychiatry. 2010;71(12):1689-1695.

10. Fagiolini A, Kupfer DJ, Rucci P, Scott JA, Novick DM, Frank E. Suicide attempts and ideation in patients with bipolar I disorder. J Clin Psychiatry. 2004;65(4):509-514.

11. Hauser M, Galling B, Correll CU. Suicidal ideation and suicide attempts in children and adolescents with bipolar disorder: a systematic review of prevalence and incidence rates, correlates, and targeted interventions. Bipolar Disord. 2013;15(5):507-523.

12. Post RM, Leverich GS, Kupka RW, et al. Early-onset bipolar disorder and treatment delay are risk factors for poor outcome in adulthood. J Clin Psychiatry. 2010;71(7):864-872.

13. Smoller JW, Finn CT. Family, twin, and adoption studies of bipolar disorder. Am J Med Genet C Semin Med Genet. 2003;123C(1):48-58.

14. Wray NR, Gottesman II. Using summary data from the Danish national registers to estimate heritabilities for schizophrenia, bipolar disorder, and major depressive disorder. Front Genet. 2012;3:118.

15. Aas M, Etain B, Bellivier F, et al. Additive effects of childhood abuse and cannabis abuse on clinical expressions of bipolar disorders. Psychol Med. Epub September 13, 2013.
16. Brown AS. The environment and susceptibility to schizophrenia. Prog Neurobiol. 2011;93(1):23-58.

17. Meli G, Ottl B, Paladini A, Cataldi L. Prenatal and perinatal risk factors of schizophrenia. J Matern Fetal Neonatal Med. 2012;25(12): 2559-2563.

18. Canetta SE, Brown AS. Prenatal infection, maternal immune activation, and risk for schizophrenia. Transl Neurosci. 2012;3(4):320-327.

19. Khandaker GM, Zimbron J, Lewis G, Jones PB. Prenatal maternal infection, neurodevelopment and adult schizophrenia: a systematic review of population-based studies. Psychol Med. 2013;43(2): 239-257.

20. Haukvik UK, McNeil T, Lange EH, et al. Pre- and perinatal hypoxia associated with hippocampus/amygdala volume in bipolar disorder. Psychol Med. 2013;27:1-11.

21. Kenny EM, Cormican P, Furlong S, et al. Excess of rare novel lossof-function variants in synaptic genes in schizophrenia and autism spectrum disorders. Mol Psychiatry. Epub October 15, 2013.

22. Craddock N, Sklar P. Genetics of bipolar disorder. Lancet. 2013;381(9878):1654-1662.

23. Szczepankiewicz A. Evidence for single nucleotide polymorphisms and their association with bipolar disorder. Neuropsychiatr Dis Treat. 2013;9:1573-1582.

24. Sklar P, Smoller JW, Fan J, et al. Whole-genome association study of bipolar disorder. Mol Psychiatry. 2008;13(6):558-569.

25. Ferreira MA, O`Donovan MC, Meng YA, et al. Wellcome Trust Case Control Consortium. Collaborative genome-wide association analysis supports a role for ANK3 and CACNA1C in bipolar disorder. Nat Genet. 2008;40(9):1056-1058.

26. Moskvina V, Craddock N, Holmans P, et al. Gene-wide analyses of genome-wide association data sets: evidence for multiple common risk alleles for schizophrenia and bipolar disorder and for overlap in genetic risk. Mol Psychiatry. 2009;14(3):252-260.

27. Kloiber S, Czamara D, Karbalai N, et al. ANK3 and CACNA1C: missing genetic link for bipolar disorder and major depressive disorder in two German case-control samples. J Psychiatr Res. 2012;46(8):973-979.

28. Zhang J, Cai J, Zhang X, et al. Does the bipolar disorder-associated CACNA1C gene confer susceptibility to schizophrenia in Han Chinese? J Mol Neurosci. 2013;51(2):474-477.

29. Zhang X, Zhang C, Wu Z, et al. Association of genetic variation in CACNA1C with bipolar disorder in Han Chinese. J Affect Disord. 2013;150(2):261-265.

30. Gershon ES, Grennan K, Busnello J, et al. A rare mutation of CACNA1C in a patient with bipolar disorder, and decreased gene expression associated with a bipolar-associated common SNP of CACNA1C in brain. Mol Psychiatry. Epub August 27, 2013.

31. Bigos KL, Mattay VS, Callicott JH, et al. Genetic variation in CACNA1C affects brain circuitries related to mental illness. Arch Gen Psychiatry. 2010;67(9):939-945.

32. Wang F, McIntosh AM, He Y, Gelernter J, Blumberg HP. The association of genetic variation in CACNA1C with structure and function of a frontotemporal system. Bipolar Disord. 2011;13(7-8):696-700.

33. Soeiro-de-Souza MG, Otaduy MC, Dias CZ, Bio DS, Machado-Vieira R, Moreno RA. The impact of the CACNA1C risk allele on limbic structures and facial emotions recognition in bipolar disorder subjects and healthy controls. J Affect Disord. 2012;141(1):94-101.

34. Soeiro-de-Souza MG, Bio DS, Dias VV, Vieta E, Machado-Vieira R, Moreno RA. The CACNA1C risk allele selectively impacts on executive function in bipolar type I disorder. Acta Psychiatr Scand. 2013;128(5):362-369.

35. Zhang Q, Shen Q, Xu Z, et al. The effects of CACNA1C gene polymorphism on spatial working memory in both healthy controls and patients with schizophrenia or bipolar disorder. Neuropsychopharmacology. 2012;37(3):677-684.

36. Paulus FM, Bedenbender J, Krach S, et al. Association of rs 1006737 in CACNA1C with alterations in prefrontal activation and frontohippocampal connectivity. Hum Brain Mapp. Epub February 13, 2013. 
37. Cross-Disorder Group of the Psychiatric Genomics Consortium, Smoller JW, Craddock N, et al. Identification of risk loci with shared effects on five major psychiatric disorders: a genome-wide analysis. Lancet. 2013;381(9875):1371-1379.

38. Green EK, Hamshere M, Forty L, et al. Replication of bipolar disorder susceptibility alleles and identification of two novel genome-wide significant associations in a new bipolar disorder case-control sample. Mol Psychiatry. 2013;18(12):1302-1307.

39. Hamshere ML, Walters JT, Smith R, et al. Genome-wide significant associations in schizophrenia to ITIH3/4, CACNA1C and SDCCAG8, and extensive replication of associations reported by the Schizophrenia PGC. Mol Psychiatry. 2013;18(6):708-712.

40. Splawski I, Timothy KW, Sharpe LM, et al. Ca(V)1.2 calcium channel dysfunction causes a multisystem disorder including arrhythmia and autism. Cell. 2004;119(1):19-31.

41. Hsiao PY, Tien HC, Lo CP, Juang JM, Wang YH, Sung RJ. Gene mutations in cardiac arrhythmias: a review of recent evidence in ion channelopathies. Appl Clin Genet. 2013;6:1-13.

42. Boczek NJ, Best JM, Tester DJ, et al. Exome sequencing and systems biology converge to identify novel mutations in the L-type calcium channel, CACNA1C, linked to autosomal dominant long QT syndrome. Circ Cardiovasc Genet. 2013;6(3):279-289.

43. Brugada J, Brugada P. Further characterization of the syndrome of right bundle branch block, ST segment elevation, and sudden cardiac death J Cardiovasc Electrophysiol. 1997;8(3):325-331.

44. Dao DT, Mahon PB, Cai X, et al. Mood disorder susceptibility gene CACNA1C modifies mood-related behaviors in mice and interacts with sex to influence behavior in mice and diagnosis in humans. Biol Psychiatry. 2010;68(9):801-810.

45. PubMed [homepage on the internet]. Available from: http://www.ncbi nlm.nih.gov/pubmed/. Accessed January 10, 2014.

46. Psychiatric GWAS Consortium Bipolar Disorder Working Group. Large-scale genome-wide association analysis of bipolar disorder identifies a new susceptibility locus near ODZ4. Nat Genet. 2011;43(10) 977-983.

47. Tesli M, Egeland R, Sønderby IE, et al. No evidence for association between bipolar disorder risk gene variants and brain structural phenotypes. J Affect Disord. 2013;151(1):291-297.

48. Heinrich A, Lourdusamy A, Tzschoppe J, et al. The risk variant in ODZ4 for bipolar disorder impacts on amygdala activation during reward processing. Bipolar Disord. Epub April 24, 2013.

49. Tesli M, Skatun KC, Ousdal OT, et al. CACNA1C risk variant and amygdala activity in bipolar disorder, schizophrenia and healthy controls. PLoS One. 2013;8(2):e56970.

50. Krug A, Witt SH, Backes H, et al. A genome-wide supported variant in CACNA1C influences hippocampal activation during episodic memory encoding and retrieval. Eur Arch Psychiatry Clin Neurosci. Epub July 17, 2013.

51. Ben-Zur T, Feige E, Motro B, Wides R. The mammalian Odz gene family: homologs of a Drosophila pair-rule gene with expression implying distinct yet overlapping developmental roles. Dev Biol. 2000;217(1): $107-120$

52. Zhou XH, Brandau O, Feng K, et al. The murine Ten-m/Odz genes show distinct but overlapping expression patterns during development and in adult brain. Gene Expr Patterns. 2003;3(4):397-405.

53. Suzuki N, Fukushi M, Kosaki K, et al. Teneurin-4 is a novel regulator of oligodendrocyte differentiation and myelination of small-diameter axons in the CNS. $J$ Neurosci. 2012;32(34):11586-11599.

54. Cichon S, Mühleisen TW, Degenhardt FA, et al. Genome-wide association study identifies genetic variation in neurocan as a susceptibility factor for bipolar disorder. Am J Hum Genet. 2011;88(3):372-381.

55. Oruč L, Kapur-Pojskić L, Ramić J, Pojskić N, Bajrović K. Assessment of relatedness between neurocan gene as bipolar disorder susceptibility locus and schizophrenia. Bosn J Basic Med Sci. 2012;12(4):245-248.

56. Mühleisen TW, Mattheisen M, Strohmaier J, et al. Association between schizophrenia and common variation in neurocan (NCAN), a genetic risk factor for bipolar disorder. Schizophr Res. 2012;138(1):69-73.
57. Schultz CC, Mühleisen TW, Nenadic I, et al. Common variation in NCAN, a risk factor for bipolar disorder and schizophrenia, influences local cortical folding in schizophrenia. Psychol Med. Epub June 24, 2013.

58. Zhou XH, Brakebusch C, Matthies H, et al. Neurocan is dispensable for brain development. Mol Cell Biol. 2001;21(17):5970-5978.

59. Rauch U, Zhou XH, Roos G. Extracellular matrix alterations in brains lacking four of its components. Biochem Biophys Res Commun. 2005;328(2):608-617.

60. Lu Y, Feskens EJ, Boer JM, et al. Exploring genetic determinants of plasma total cholesterol levels and their predictive value in a longitudinal study. Atherosclerosis. 2010;213(1):200-205.

61. Yan TT, Yin RX, Li Q, et al. Sex-specific association of rs 16996148 SNP in the NCAN/CILP2/PBX4 and serum lipid levels in the Mulao and Han populations. Lipids Health Dis. 2011;10:248.

62. Zhou L, Ding H, Zhang X, et al. Genetic variants at newly identified lipid loci are associated with coronary heart disease in a Chinese Han population. PLoS One. 2011;6(11):e27481.

63. Speliotes EK, Yerges-Armstrong LM, Wu J, et al. Genome-wide association analysis identifies variants associated with nonalcoholic fatty liver disease that have distinct effects on metabolic traits. PLoS Genet. 2011;7(3):e1001324.

64. Gorden A, Yang R, Yerges-Armstrong LM, et al. Genetic variation at NCAN locus is associated with inflammation and fibrosis in nonalcoholic fatty liver disease in morbid obesity. Hum Hered. 2013;75(1): 34-43.

65. Nakayama K, Bayasgalan T, Yamanaka K, et al. Large scale replication analysis of loci associated with lipid concentrations in a Japanese population. J Med Genet. 2009;46(6):370-374.

66. Online Mendelian Inheritance in Man (OMIM) [homepage on the internet]. Available from: http://www.ncbi.nlm.nih.gov/omim. Accessed January 10, 2014.

67. Boer H, Holland A, Whittington J, Butler J, Webb T, Clarke D. Psychotic illness in people with Prader Willi syndrome due to chromosome 15 maternal uniparental disomy. Lancet. 2002;359(9301):135-136.

68. Burnside RD, Pasion R, Mikhail FM, et al. Microdeletion/ microduplication of proximal 15q11.2 between BP1 and BP2: a susceptibility region for neurological dysfunction including developmental and language delay. Hum Genet. 2011;130(4):517-528.

69. Skokauskas N, Sweeny E, Meehan J, Gallagher L. Mental health problems in children with Prader-Willi syndrome. J Can Acad Child Adolesc Psychiatry. 2012;21(3):194-203.

70. Fan Z, Greenwood R, Fisher A, Pendyal S, Powell CM. Characteristics and frequency of seizure disorder in 56 patients with Prader-Willi syndrome. Am J Med Genet A. 2009;149A(7):1581-1584.

71. Sutcliffe JS, Nakao M, Christian S, et al. Deletions of a differentially methylated $\mathrm{CpG}$ island at the SNRPN gene define a putative imprinting control region. Nat Genet. 1994;8(1):52-58.

72. Hall BD, Smith DW. Prader-Willi syndrome. A resumé of 32 cases including an instance of affected first cousins, one of whom is of normal stature and intelligence. J Pediatr. 1972;81(2):286-293.

73. Autio S, Pihko H, Tengström C. Clinical features in a de novo interstitial deletion 15q13 to q15. Clin Genet. 1988;34(5):293-298.

74. Ninomiya S, Yokoyama Y, Kawakami M, Une T, Maruyama H, Morishima T. Unique maternal deletion of $15 \mathrm{q}$ in a patient with some symptoms of Prader-Willi syndrome. Pediatr Int. 2005;47(5):541-545.

75. Sharp AJ, Mefford HC, Li K, et al. A recurrent 15q13.3 microdeletion syndrome associated with mental retardation and seizures. Nat Genet. 2008;40(3):322-328.

76. Helbig I, Mefford HC, Sharp AJ, et al. 15q13.3 microdeletions increase risk of idiopathic generalized epilepsy. Nat Genet. 2009;41(2):160-162.

77. de Kovel CG, Trucks H, Helbig I, et al. Recurrent microdeletions at $15 \mathrm{q} 11.2$ and $16 \mathrm{p} 13.11$ predispose to idiopathic generalized epilepsies. Brain. 2010;133(Pt 1):23-32.

78. van Bon BW, Mefford HC, Menten B, et al. Further delineation of the $15 \mathrm{q} 13$ microdeletion and duplication syndromes: a clinical spectrum varying from non-pathogenic to a severe outcome. $J$ Med Genet. 2009;46(8):511-523. 
79. Miller DT, Shen Y, Weiss LA, et al. Microdeletion/duplication at $15 q 13.2 q 13.3$ among individuals with features of autism and other neuropsychiatric disorders. J Med Genet. 2009;46(4):242-248.

80. Pagnamenta AT, Wing K, Sadighi Akha E, et al. A 15q13.3 microdeletion segregating with autism. Eur J Hum Genet. 2009;17(5):687-692.

81. Ben-Shachar S, Lanpher B, German JR, et al. Microdeletion 15q13.3: a locus with incomplete penetrance for autism, mental retardation, and psychiatric disorders. J Med Genet. 2009;46(6):382-388.

82. Sahoo T, Theisen A, Rosenfeld JA, et al. Copy number variants of schizophrenia susceptibility loci are associated with a spectrum of speech and developmental delays and behavior problems. Genet Med. 2011;13(10):868-880.

83. Shinawi M, Schaaf CP, Bhatt SS, et al. A small recurrent deletion within 15 q13.3 is associated with a range of neurodevelopmental phenotypes. Nat Genet. 2009;41(12):1269-1271.

84. Hoppman-Chaney N, Wain K, Seger PR, Superneau DW, Hodge JC. Identification of single gene deletions at 15q13.3: further evidence that CHRNA7 causes the 15q13.3 microdeletion syndrome phenotype. Clin Genet. 2013;83(4):345-351.

85. Endris V,Hackmann K, Neuhann TM, et al. Homozygous loss of CHRNA7 on chromosome $15 \mathrm{q} 13.3$ causes severe encephalopathy with seizures and hypotonia. Am J Med Genet A. 2010;152A(11):2908-2911.

86. GeneReviews ${ }^{\mathrm{TM}}$ [internet]. Edited by Roberta A Pagon, Editor-in-chief, Margaret P Adam, Thomas D Bird, Cynthia R Dolan, Chin-To Fong, Richard JH Smith, and Karen Stephens. Seattle (WA): University of Washington, Seattle; 1993-2014. Available from http:/www.ncbi.nlm. nih.gov/books/. Accessed October 18, 2013.

87. Mehta L, Duckett DP, Young ID. Behaviour disorder in monosomy 10qter. J Med Genet. 1987;24(3):185-186.

88. Courtens W, Wuyts W, Rooms L, Pera SB, Wauters J. A subterminal deletion of the long arm of chromosome 10: a clinical report and review. Am J Med Genet A. 2006;140(4):402-409.

89. Miller ND, Nance MA, Wohler ES, et al. Molecular (SNP) analyses of overlapping hemizygous deletions of 10q25.3 to 10qter in four patients: evidence for HMX2 and HMX3 as candidate genes in hearing and vestibular function. Am J Med Genet A. 2009;149A(4):669-680.

90. Yatsenko SA, Kruer MC, Bader PI, et al. Identification of critical regions for clinical features of distal 10q deletion syndrome. Clin Genet. 2009;76(1):54-62.

91. Irving $\mathrm{M}$, Hanson $\mathrm{H}$, Turnpenny $\mathrm{P}$, et al. Deletion of the distal long arm of chromosome 10; is there a characteristic phenotype? A report of $15 \mathrm{de}$ novo and familial cases. Am J Med Genet A. 2003;123A(2):153-163.
92. Bassett AS, Chow EW, Husted J, et al. Clinical features of 78 adults with 22q11 deletion syndrome. Am J Med Genet A. 2005;138(4):307-313.

93. McLean SD, Saal HM, Spinner NB, Emanuel BS, Driscoll DA. Velo-cardio-facial syndrome. Intrafamilial variability of the phenotype. Am J Dis Child. 1993;147(11):1212-1216.

94. Jolin EM, Weller RA, Weller EB. Occurrence of affective disorders compared to other psychiatric disorders in children and adolescents with 22q11.2 deletion syndrome. J Affect Disord. 2012;136(3):222-228.

95. Aneja A, Fremont WP, Antshel KM, et al. Manic symptoms and behavioral dysregulation in youth with velocardiofacial syndrome (22q11.2 deletion syndrome). J Child Adolesc Psychopharmacol. 2007;17(1):105-114.

96. Shprintzen RJ, Goldberg RB, Young D, Wolford L. The velo-cardio-facial syndrome: a clinical and genetic analysis. Pediatrics. 1981;67(2):167-172.

97. Shprintzen RJ. Velo-cardio-facial syndrome: 30 Years of study. Dev Disabil Res Rev. 2008;14(1):3-10.

98. Evers LJ, Vermaak MP, Engelen JJ, Curfs LM. The velocardiofacial syndrome in older age: dementia and autistic features. Genet Couns. 2006;17(3):333-340.

99. Butcher NJ, Chow EW, Costain G, Karas D, Ho A, Bassett AS. Functional outcomes of adults with 22q11.2 deletion syndrome. Genet Med. 2012;14(10):836-843.

100. De Leersnyder H. Smith-Magenis syndrome. Handb Clin Neurol. 2013;111:295-2966.

101. Girirajan S, Elsas LJ 2nd, Devriendt K, Elsea SH. RAI1 variations in Smith-Magenis syndrome patients without $17 \mathrm{p} 11.2$ deletions. J Med Genet. 2005;42(11):820-828.

102. Elsea SH, Williams SR. Smith-Magenis syndrome: haploinsufficiency of RAI1 results in altered gene regulation in neurological and metabolic pathways. Expert Rev Mol Med. 2011;13:e14.

103. Juyal RC, Figuera LE, Hauge X, et al. Molecular analyses of $17 \mathrm{p} 11.2$ deletion in 62 Smith-Magenis syndrome patients. Am J Med Genet. 1996;58:998-1007.

104. Gershon ES, Alliey-Rodriguez N. New ethical issues for genetic counseling in common mental disorders. Am J Psychiatry. 2013;170(9): 968-976.

105. Wilfond BS, Thomson EJ. Models of public health genetic policy development. In: Khoury MJ, Burke W, Thomson EJ, editors. Genetics and Public Health in the 21th Century. Oxford, UK: Oxford University Press; 2000:61-81.
The Application of Clinical Genetics

\section{Publish your work in this journal}

The Application of Clinical Genetics is an international, peer-reviewed open access journal that welcomes laboratory and clinical findings in the field of human genetics. Specific topics include: Population genetics; Functional genetics; Natural history of genetic disease; Management of genetic disease; Mechanisms of genetic disease; Counseling and ethical

\section{Dovepress}

issues; Animal models; Pharmacogenetics; Prenatal diagnosis; Dysmorphology. The manuscript management system is completely online and includes a very quick and fair peer-review system, which is all easy to use. Visit http://www.dovepress.com/testimonials.php to read real quotes from published authors. 